\title{
A linear programming reformulation of the standard quadratic optimization problem
}

\author{
E. de Klerk • D. V. Pasechnik
}

Received: 31 March 2006/Accepted: 21 April $2006 /$

Published online: 21 July 2006

(C) Springer Science+Business Media B.V. 2006

\begin{abstract}
The problem of minimizing a quadratic form over the standard simplex is known as the standard quadratic optimization problem (SQO). It is NP-hard, and contains the maximum stable set problem in graphs as a special case. In this note, we show that the SQO problem may be reformulated as an (exponentially sized) linear program (LP). This reformulation also suggests a hierarchy of polynomial-time solvable LP's whose optimal values converge finitely to the optimal value of the SQO problem. The hierarchies of LP relaxations from the literature do not share this finite convergence property for SQO, and we review the relevant counterexamples.
\end{abstract}

Keywords Linear programming • Standard quadratic optimization · Positive polynomials

\section{Introduction}

The standard quadratic optimization (SQO) problem is to find the global minimizers of a quadratic form over the standard simplex, i.e. we consider the global optimization problem

$$
\underline{p}:=\min _{x \in \Delta_{n}} x^{T} Q x,
$$

where $Q \in \mathcal{S}_{n}$ (the space of symmetric $n \times n$ matrices), and $\Delta_{n}$ is the standard simplex in $\mathbb{R}^{n}$, namely

E. de Klerk $(\varangle)$

Department of Econometrics and Operations Research, Faculty of Economics and Business Administration, Tilburg University, P.O. Box 90153, 5000 Tilburg, The Netherlands

e-mail: E.deKlerk@uvt.nl

D. V. Pasechnik

School of Physical and Mathematical Sciences, Nanyang Technological University, 1 Nanyang Walk, Blk 5 Singapore 637616

e-mail: dima@ntu.edu.sg 


$$
\Delta_{n}=\left\{x \in \mathbb{R}^{n}: \sum_{i=1}^{n} x_{i}=1, x \geq 0\right\}
$$

Problem (1) can be rewritten as follows:

$$
\begin{aligned}
\underline{p} & =\max _{\lambda \in \mathbb{R}}\left\{\lambda: x^{T} Q x-\lambda \geq 0 \quad \forall x \in \Delta_{n}\right\} \\
& =\max _{\lambda \in \mathbb{R}}\left\{\lambda: x^{T}\left(Q-\lambda e e^{T}\right) x \geq 0 \quad \forall x \in \Delta_{n}\right\} \\
& =\max _{\lambda \in \mathbb{R}}\left\{\lambda: Q-\lambda e e^{T} \in \mathcal{C}_{n}\right\},
\end{aligned}
$$

where $\mathcal{C}_{n}$ denotes the cone of $n \times n$ symmetric copositive matrices:

$$
\mathcal{C}_{n}:=\left\{M \in \mathcal{S}_{n}, x^{T} M x \geq 0 \quad \forall x \in \mathbb{R}^{n}, x \geq 0\right\} .
$$

The SQO problem is NP-hard since it contains the maximum stable set problem in graphs as a special case: Motzkin and Straus [14] proved that

$$
\frac{1}{\alpha(G)}=\min _{x \in \Delta} x^{T}(A+I) x
$$

where $A$ is the adjacency matrix of a given graph $G$, and $\alpha(G)$ is the stability number (co-clique number) of $G$.

Other applications of SQO include portfolio optimization, game theory, and population dynamics problems (see the review paper by Bomze [2] and the references therein). A recent application is the estimation of crossing numbers in certain classes of graphs [7].

Although, SQO is NP-hard, it allows a polynomial time approximation scheme (PTAS). This was shown by Bomze and De Klerk [3], and a different proof was subsequently given by Nesterov [15]. This result was extended to optimization of forms of any fixed degree over $\Delta$ by De Klerk et al. [6] (see also Faybusovich [8]).

In this note, we show that the SQO problem (1) has an (exponentially sized) linear programming (LP) reformulation. This was known for the special case of computing the stability number of a graph from the work by Sherali and Adams [19], but is new for the general SQO problem to the best of our knowledge.

This result adds to the growing literature on NP-hard problems that allow exact LP or semidefinite programming (SDP) reformulations of exponential size (see Lasserre [10] and Laurent [13] for the latest results).

The LP reformulation also suggests a hierarchy of LP approximations of (1) with optimal values that converge finitely to the optimal value $p$ of (1) from above. We compare this to two convergent hierarchies of LP approximations from the literature. The first is based on a theorem by Polyá on forms positive on the simplex, and was studied by several authors $[3,5,6,8,9,16,20]$. The second employs a representation theorem by Krivine and others, and was introduced by Lasserre [11, 12].

Both these hierarchies give sequences of lower bounds that converge to $p$, but the convergence is not finite in general. We will review relevant counterexamp̄es from the literature in Sect. 6. 


\section{Notation}

- $A_{J K}$ : submatrix of $A$ with rows indexed by the (nonempty) index set $J$ and columns by the (nonempty) index set $K$.

- If $\alpha \in \mathbb{Z}_{+}^{n}$ and $x \in \mathbb{R}^{n}$ then $|\alpha|:=\sum_{i=1}^{n} \alpha_{i}$ and $x^{\alpha}:=\prod_{i=1}^{n} x_{i}^{\alpha_{i}}$.

- If $\alpha, \beta \in \mathbb{Z}_{+}^{n}$, then $\alpha \preceq \beta$ means $\alpha_{i} \leq \beta_{i}(i=1, \ldots, n)$.

- $I_{n}$ : identity matrix of size $n \times n$ (or of size determined by the context if the subscript is omitted).

- $e_{n}$ all-ones vector of size $n$ (or of size determined by the context if the subscript is omitted).

- If $A \in \mathcal{S}_{n}, A \succeq 0$ ( $\left.A \preceq 0\right)$ means $A$ is positive semi- definite (negative semidefinite).

\section{A characterization of matrix copositivity}

The following theorem gives a characterization of copositive matrices. We include a proof for completeness.

Theorem 1 (Gaddum [4]) If $M \in \mathcal{S}_{n}$, the following two statements are equivalent:

(a) $M$ is copositive;

(b) For all $J \subseteq\{1, \ldots, n\}, J \neq \emptyset$, the following system has a solution:

$$
M_{J J} x_{J} \geq 0, \quad x_{J} \geq 0, \quad e_{|J|}^{T} x_{J}=1 .
$$

Proof Proof of (a) $\Longrightarrow(\mathrm{b})$ :

Assume that $M$ is copositive, and let $I=\{1, \ldots, n\}$. By the Farkas lemma, the system (3) has no solution if and only if the following system has a solution:

$$
M y \leq-e, \quad y \geq 0 .
$$

Since $y \neq 0$, one has $y^{T} M y \leq-e^{T} y<0$, a contradiction.

Proof of $(\mathrm{b}) \Longrightarrow(\mathrm{a})$ :

The proof is by induction on $n$; the case $n=1$ is trivial, so assume that $n>1$ and that the required result holds for all matrices of order less than $n$. These assumptions imply that the system (3) has a solution for any $J \subseteq\{1, \ldots, n\}$, and that $M_{J J}$ is copositive if $|J|<n$.

Let $\bar{x}$ be the solution of (3) corresponding to $J=\{1, \ldots, n\}$, and let $x \geq 0$ be given. Let $\lambda \geq 0$ be such that $x-\lambda \bar{x} \geq 0$ but $x-\lambda \bar{x} \ngtr 0$. Now

$$
x^{T} M x=(x-\lambda \bar{x})^{T} M(x-\lambda \bar{x})+\lambda(2 x-\lambda \bar{x})^{T} M \bar{x} .
$$

The right-hand side terms are both nonnegative since all proper principal submatrices of $M$ are copositive by assumption, $2 x-\lambda \bar{x} \geq 0$, and $M \bar{x} \geq 0$. 


\section{LP reformulation of standard quadratic optimization}

Using Theorem 1, we may rewrite the copositivity requirement $Q-\lambda e e^{T} \in \mathcal{C}_{n}$ as the requirement that, for all $J \subseteq\{1, \ldots, n\}, J \neq \emptyset$, the following system has a solution:

$$
\left(Q-\lambda e e^{T}\right)_{J J} x_{J} \geq 0, \quad x_{J} \geq 0, e_{|J|}^{T} x_{J}=1 .
$$

Using $e_{|J|}^{T} x_{J}=1$, this system is the same as

$$
Q_{J J} x_{J}-\lambda e_{|J|} \geq 0, \quad x_{J} \geq 0, \quad e_{|J|}^{T} x_{J}=1 .
$$

Thus we obtain the following LP reformulation of (1):

$$
\underline{p}=\max \left\{\lambda: Q_{J J} x_{J}-\lambda e_{|J|} \geq 0, x_{J} \geq 0, e_{|J|}^{T} x_{J}=1 \quad \forall J \subseteq\{1, \ldots, n\}\right\} .
$$

Note that this is an LP where the number of variables is:

$$
1+\sum_{r=1}^{n} r\left(\begin{array}{l}
n \\
r
\end{array}\right)=1+\frac{1}{2} n 2^{n}
$$

The number of inequality constraints is $2 \sum_{r=1}^{n} r\left(\begin{array}{l}n \\ r\end{array}\right)=n 2^{n}$ (including the nonnegativity of the variables), and there are $2^{n}-1$ equality constraints.

Example 1 Consider the maximum stable set problem:

$$
\frac{1}{\alpha(G)}=\min _{x \in \Delta} x^{T}(A+I) x,
$$

where $A$ is the adjacency matrix of a given graph $G$, and $\alpha(G)$ is the stability number (co-clique number) of $G$.

The copositive programming reformulation is

$$
\max _{\lambda \in \mathbb{R}}\left\{\lambda: A+I-\lambda e e^{T} \in \mathcal{C}_{n}\right\}
$$

and the LP reformulation is

$$
\frac{1}{\alpha(G)}=\max \left\{\lambda: A_{J J} x_{J}+x_{J}-\lambda e_{|J|} \geq 0, x_{J} \in \Delta_{|J|} \quad \forall J \subseteq\{1, \ldots, n\}\right\} .
$$

An optimal solution of the LP reformulation is obtained by choosing $\lambda=\frac{1}{\alpha(G)}$ and $x_{J} \in \Delta_{|J|}$ as the normalized incidence vector of any maximum stable set $S_{J}$ in the subgraph induced by the vertices in $J$ for each $J \subseteq\{1, \ldots, n\}$.

In this case we have $A_{J J} x_{J}+x_{J} \geq \frac{1}{\left|S_{J}\right|} e_{|J|} \geq \frac{1}{\alpha(G)} e_{|J|}$, as required.

\section{Relation to the KKT conditions}

Since problem (1) satisfies the regularity condition that the active constraint gradients are always linearly independent, the KKT conditions are necessary for optimality (cf. Theorem 4.3.7 in [1]). The KKT optimality conditions are given by:

$$
Q x \geq \lambda e, \quad x \in \Delta_{n}
$$


as well as

$$
x^{T} Q x=\lambda .
$$

If $\bar{x} \in \Delta_{n}$ satisfies $Q \bar{x} \geq\left(\bar{x}^{T} Q \bar{x}\right) e$, then we call $\bar{x}$ a $K K T$ point of problem (1).

The conditions (5) and (6) imply the complementarity condition:

$$
x_{i}\left((Q x)_{i}-x^{T} Q x\right)=0, \quad i=1, \ldots, n .
$$

Note that the conditions (5) form a subset of the constraint set of the LP reformulation (4), corresponding to $J=\{1, \ldots, n\}$.

Note that we may rewrite (4) as

$$
\underline{p}=\min _{J \subseteq\{1, \ldots, n\}, J \neq \emptyset} t_{J},
$$

where

$$
t_{J}:=\max \left\{t: Q_{J J} x_{J}-t e_{|J|} \geq 0, x_{J} \in \Delta_{|J|}\right\} .
$$

The inner maximization problems are related to the KKT conditions of the SQO problems obtained by restricting the optimization in (1) to a specific face of $\Delta_{n}$, namely the face obtained by setting $x_{i}=0$ if $i \notin J$ :

$$
\min \left\{x_{J}^{T} Q_{J J} x_{J}: x_{J} \in \Delta_{|J|}\right\} .
$$

Lemma 1 If problem (9) has a positive KKT point $x_{J}>0$, then the optimal value of problem (8) is $t_{J}=x_{J}^{T} Q x_{J}$.

Proof Since $x_{J}$ is a KKT point it satisfies the complementarity condition (7). Using $x_{J}>0$ this reduces to $Q_{J J} x_{J}=\left(x_{J}^{T} Q_{J J} x_{J}\right) e$.

The dual of problem (8) is

$$
\min \left\{t: Q_{J J} y-t e_{|J|} \leq 0, y \in \Delta_{|J|}\right\} .
$$

Note that $t=x_{J}^{T} Q_{J J} x_{J}$ and $y=x_{J}$ is a feasible solution to this problem. Since it is also feasible to the primal problem (8) with the same objective value, it is an optimal solution to both problems.

The values $t_{J}$ in (8) do not always correspond to objective values at KKT points, though, as the next example shows.

Example 2 Let

$$
Q=\left[\begin{array}{ll}
2 & 1 \\
1 & \frac{1}{2}
\end{array}\right]
$$

Now $\frac{1}{2}=\underline{p}:=\min _{x \in \Delta_{2}} x^{T} Q x$, and the unique global minimizer is $x^{*}=[0,1]^{T}$. This is also the unique KKT point, since $Q \succeq 0$, i.e. we have a convex optimization problem. However, one has

$$
t_{\{1,2\}}=\max \left\{t: Q x \geq t e_{2}, x \in \Delta_{2}\right\}=1,
$$

which corresponds to $x=[1,0]^{T}$.

Theorem 2 Assume that $x^{*}$ is a global minimizer of the SQO problem (1) and the support of $x^{*}$ is $J$. 
Then, if $\left(t_{J}, x_{J}\right)$ is an optimal solution of (8), one has $t_{J}=p$, and $x_{J}$ defines an optimal solution of (1).

Proof If $x^{*}$ is a global minimizer of (1) with support $J$, then the vector $x_{J}^{*} \in \Delta_{|J|}$ formed by the positive components of $x^{*}$ is a global minimizer of (9). Thus $x_{J}^{*}>0$ is also a KKT point of (9), and the required result follows from Lemma 1.

Example 3 Let

$$
Q=\left[\begin{array}{rrr}
0 & 0 & 1 \\
0 & 0 & -1 \\
1 & -1 & 0
\end{array}\right]
$$

Now $-\frac{1}{2}=p:=\min _{x \in \Delta_{3}} x^{T} Q x$, and the global minimizer is $x^{*}=\left[0, \frac{1}{2}, \frac{1}{2}\right]^{T}$. The problem also has other KKT points, namely all points of the form

$$
x=[\alpha,(1-\alpha), 0]^{T}, \quad \alpha \in[0,1] .
$$

It is easy to verify that

$$
t_{\{1,2,3\}}=0, \quad t_{\{1,2\}}=t_{\{1,3\}}=0, \quad t_{\{2,3\}}=-\frac{1}{2}, \quad t_{\{1\}}=t_{\{2\}}=t_{\{3\}}=0 .
$$

Thus $\underline{p}=\min _{J \subseteq\{1,2,3\}} t_{J}=-\frac{1}{2}$. Since the support of the global minimizer $x^{*}$ is $\{2,3\}$, the minimum $\underline{p}$ corresponds to $t_{\{2,3\}}$.

\section{A hierarchy of LP relaxations}

One can define a hierarchy of LP relaxations that approximate (1) as follows:

$$
\underline{p}^{(r)}=\max \left\{\lambda: Q_{J J} x_{J}-\lambda e_{|J|} \geq 0, x_{J} \in \Delta_{|J|}, \quad \forall|J| \leq r \text { or }|J| \geq n-r+1\right\},
$$

for $r=1,2, \ldots,\left\lfloor\frac{1}{2} n\right\rfloor$.

Note that - for fixed $r$ - the number of constraints and variables are polynomial in $n$, and $p^{(r)}$ can therefore be obtained in polynomial time.

We can summarize our main results in the following theorem.

Theorem 3 Let $p$ denote the optimal value of problem (1) as before, and define $p^{(r)}$ as in (10) for $r=1,2, \ldots,\left\lfloor\frac{1}{2} n\right\rfloor$. One has $\underline{p}^{(r)} \geq \underline{p}\left(r=1,2, \ldots,\left\lfloor\frac{1}{2} n\right\rfloor\right)$ with equality in the following cases:

1 problem (1) has an optimal solution with support of cardinality at most $r$ or at least $n-r$

$2 \quad r=\left\lfloor\frac{1}{2} n\right\rfloor ;$

$3 Q \preceq 0$ and $r \geq 1$;

$4 Q=A+I$ where $A$ is the adjacency matrix of a graph $G$ and $r \geq \min \{\alpha(G), n-\alpha(G)\}$.

Proof Item 1 follows from Theorem 2, and item 2 is a consequence of item 1.

In item 3 , the objective is concave since $Q \preceq 0$ and the global minimum is therefore, attained at an extreme point of the simplex, i.e. at a standard unit vector. In particular, it follows that $p=\min _{i} Q_{i i}$. By considering index sets $J=\{i\}$ in (10), we get the inequalities $\underline{p}^{(1)} \leq Q_{i i}(i=1, \ldots, n)$. Since we know that $\underline{p}^{(1)} \geq \underline{p}$, the result follows. 
The result in item 4 follows from Theorem 2, since each global minimizer of problem (2) is the normalized incidence vector of a maximum stable set.

\section{Relation to existing LP approximations}

In this section, we compare the hierarchy of LP relaxations (10) of the previous section to two hierarchies from the literature.

\subsection{Relaxation using Polyá's theorem}

Polyá [17] gave the following representation theorem for polynomials positive on the simplex (see also [18]).

Theorem 4 (Polyá) If a homogeneous polynomial (form) $p$ is positive on $\Delta_{n}$, then the form

$$
p(x)\left(\sum_{i=1}^{n} x_{i}\right)^{r}
$$

only has nonnegative coefficients if $r$ is sufficiently large.

This suggests the following polynomial-time LP approximations of (1):

$$
\rho^{(r)}:=\max t
$$

such that

$$
\left(x^{T} Q x-t\left(e^{T} x\right)^{2}\right)\left(\sum_{i=1}^{n} x_{i}\right)^{r} \text { only has nonnegative coefficients. }
$$

Note that, for fixed $r$, this problem may easily be reformulated as an LP with one variable and number of constraints polynomial in $n$.

Indeed, if $p(x)=\sum_{\alpha} a_{\alpha} x^{\alpha}$ has degree $d$, then the coefficient $A_{\beta}$ of $x^{\beta}$ in $p(x)$ $\left(\sum_{i=1}^{n} x_{i}\right)^{r}$ is given by

$$
A_{\beta}=\sum_{|a|=d, \alpha \preceq \beta}\left(\frac{r !}{\prod_{i=1}^{n}\left(\beta_{i}-\alpha_{i}\right) !}\right) a_{\alpha} .
$$

Thus the coefficients of $\left(x^{T} Q x-t\left(e^{T} x\right)^{2}\right)\left(\sum_{i=1}^{n} x_{i}\right)^{r}$ depend linearly on the coefficients of $\left(x^{T} Q x-t\left(e^{T} x\right)^{2}\right)$, which in turn depend linearly on $t$.

One has $\rho^{(r)} \leq \underline{p}$, and, by Polyá's theorem, $\rho^{(r)} \rightarrow \underline{p}$ as $r \rightarrow \infty$.

Bomze and De Klerk [3] showed that this approach yields a polynomial time approximation scheme for problem (1). In particular, they showed that

$$
\underline{p}-\rho^{(r)} \leq \frac{1}{r+1}(\bar{p}-\underline{p})
$$

where $\bar{p}:=\max _{x \in \Delta_{n}} x^{T} Q x$. However, the convergence $\rho^{(r)} \rightarrow \underline{p}$ is not finite in general, as the next example shows. 
Example 4 Let

$$
Q=\left[\begin{array}{cc}
1 & -1 \\
-1 & 1
\end{array}\right]
$$

so that $\underline{p}=\min _{x \in \Delta_{n}} x^{T} Q x=0$, with global minimizer $x_{1}=x_{2}=\frac{1}{2}$.

However, one will not have $\rho^{(r)}=0$ for any finite value of $r$. Indeed, if $r$ is even, then the coefficient of the monomial $x_{1}^{\frac{1}{2} r+1} x_{2}^{\frac{1}{2} r+1}$ in $x^{T} Q x\left(\sum_{i=1}^{n} x_{i}\right)^{r}$ equals

$$
1\left(\begin{array}{c}
r \\
\frac{1}{2} r-1
\end{array}\right)+1\left(\begin{array}{c}
r \\
\frac{1}{2} r-1
\end{array}\right)-2\left(\begin{array}{c}
r \\
\frac{1}{2} r
\end{array}\right)=\frac{-(r+2) r !}{\left(\left(\frac{1}{2} r+1\right) !\right)^{2}}<0 .
$$

\subsection{Relaxation using Krivine's theorem}

The following is a special case of a theorem due to Krivine, Becker and Schwartz, Marshall, and Vasilescu (for a discussion of the general result, see Lasserre [12], and the references therein).

To simplify the presentation it will be useful to work with the standard simplex in the inequality form $\left\{x \in \mathbb{R}_{+}^{n} \mid \sum_{i=1}^{n} x_{i} \leq 1\right\}$.

Theorem 5 Assume $f \in \mathbb{R}\left[x_{1}, \ldots, x_{n}\right]$ is positive on $\left\{x \in \mathbb{R}_{+}^{n} \mid \sum_{i=1}^{n} x_{i} \leq 1\right\}$. Then there exist vectors $\alpha \in \mathbb{N}^{n+1}$ and $\beta \in \mathbb{N}^{n+1}$ such that

$$
f(x)=\sum_{\alpha, \beta} c_{\alpha \beta}\left(\sum_{i=1}^{n} x_{i}\right)^{\alpha_{0}}\left(1-\sum_{i=1}^{n} x_{i}\right)^{\beta_{0}} \prod_{i=1}^{n} x_{i}^{\alpha_{i}}\left(1-x_{i}\right)^{\beta_{i}}
$$

for finitely many positive coefficients $\left\{c_{\alpha \beta}\right\}$.

This representation theorem suggests another hierarchy of LP approximations for (1), due to Lasserre $[11,12]$. In order to apply the theorem to $(1)$, we eliminate the variable $x_{n}$ in (1) via $x_{n}=1-\sum_{i=1}^{n-1} x_{i}$ in order to work with the simplex in inequality form.

Thus we now consider (1) in the form

$$
\underline{p}=\min x^{T} A x+b^{T} x
$$

subject to $\left\{x \in \mathbb{R}_{+}^{n} \mid \sum_{i=1}^{n} x_{i} \leq 1\right\}$.

The LP approximations of Lasserre, when applied to this problem, take the form

$$
v^{(r)}:=\max t
$$

such that there exist nonnegative values $c_{\alpha \beta}$ so that

$$
x^{T} A x+b^{T} x-t=\sum_{\alpha, \beta} c_{\alpha \beta}\left(\sum_{i=1}^{n} x_{i}\right)^{\alpha_{0}}\left(1-\sum_{i=1}^{n} x_{i}\right)^{\beta_{0}} \prod_{i=1}^{n} x_{i}^{\alpha_{i}}\left(1-x_{i}\right)^{\beta_{i}}
$$

for nonnegative integer vectors $\alpha, \beta$ such that $|\alpha|+|\beta| \leq r$.

Once again, one has $v^{(r)} \leq \underline{p}$, and, by Krivine's theorem, $v^{(r)} \rightarrow \underline{p}$ as $r \rightarrow \infty$. However, this convergence is not finite in general, as the following example shows. 
Example 5 (Lasserre) $\min x^{2}-x$ subject to $0 \leq x \leq 1$. The global minimizer is $x=\frac{1}{2}$ with optimal value $p=-1 / 4$.

The LP relaxations take the form:

$$
v^{(r)}:=\max t
$$

so that

$$
x^{2}-x-t=\sum_{i+j \leq r} c_{i j} x^{i}(1-x)^{j}
$$

for some nonnegative values $c_{i j}$. Note that, for $t=-1 / 4$, the equality can never hold (look at $x=\frac{1}{2}$ ).

\section{Conclusion and discussion}

We have given an LP reformulation of the SQO problem (see (1)). This reformulation also suggests a hierarchy of polynomial-time solvable LP's whose optimal values converge finitely to the optimal value of the SQO problem. We have also reviewed the fact that the hierarchies of LP relaxations from the literature do not share the finite convergence property for SQO.

The LP problems appearing the hierarchy (10) can in practice only be solved for relatively small values of $r$. For example, for $r=20$, the LP problem (10) already has a number of variables and constraints of the order $10^{7}$.

It is therefore of interest to derive an error bound for our LP hierarchy for fixed values of $r$, similar to the error bound (12) that holds for the LP hierarchy based on Polyá's representation theorem. However, it is not clear at this time how to obtain such error bounds, or indeed, if the new hierarchy also gives a PTAS for the SQO problem.

Acknowledgements The authors are indebted to Immanuel Bomze for illuminating discussions concerning Lemma 1, and for providing reference [2], and to Jean Lasserre and Eligius Hendrix for useful comments. Supported by the Netherlands Organisation for Scientific Research grants NWO 613.000.214 and NWO 016.025.026. Part of this research was performed while the first author was on leave from the Department of Combinatorics and Optimization, University of Waterloo. This work was completed while the second author was working at Tilburg University, The Netherlands.

\section{References}

1. Bazarraa, M.S., Sherali, H.D., Shetty, C.M.: Nonlinear Programming: Theory and Algorithms. Wiley, New York (1993)

2. Bomze, I.M.: Regularity versus degeneracy in dynamics, games, and optimization: a unified approach to different aspects. SIAM Rev. 44(3), 394-414 (2002)

3. Bomze, I.M., De Klerk, E.: Solving standard quadratic optimization problems via linear, semidefinite and copositive programming. Global Optim. 24(2), 163-185 (2002)

4. Gaddum, J.W.: Linear inequalities and quadratic forms. Pacific J. Math. 8: 411-414 (1958)

5. de Klerk, E., Laurent, M., Parrilo, P.: On the equivalence of algebraic approaches to the minimization of forms on the simplex. In: Henrion, D., Garulli, A. (eds.) Positive Polynomials in Control LNCIS, vol. 312, Springer Verlag, Berlin (2005)

6. de Klerk, E., Laurent, M., Parrilo, P.: A PTAS for the minimization of polynomials of fixed degree over the simplex. Theoretical Computer Science, to appear

7. de Klerk, E., Maharry, J., Pasechnik, D.V., Richter, B., Salazar, G.: Improved bounds for the crossing numbers of $K_{m, n}$ and $K_{n}$. SIAM J. Discrete Math. 20(1), 189-202 (2006) 
8. Faybusovich, L.: Global optimization of homogeneous polynomials on the simplex and on the sphere. In Floudas, C., Pardalos, P. (eds.), Frontiers in Global Optimization. Kluwer Academic Publishers, Dordrecht (2003)

9. de Klerk, E., Pasechnik, D.V.: Approximation of the stability number of a graph via copositive programming. SIAM J. Optim. 12, 875-892 (2002)

10. Lasserre, J.B.: An explicit equivalent positive semidefinite program for nonliner 0-1 programs. SIAM J. Optim. 12, 756-769 (2002)

11. Lasserre, J.B.: Semidefinite programming vs. LP relaxations for polynomial programming. Math. Oper. Res. 27(2), 347-360 (2002)

12. Lasserre, J.B.: Polynomial programming: LP relaxations also converge. SIAM J. Optim. 15, 383393 (2004)

13. Laurent, M.: Semidefinite representations for finite varieties. Math. Program. to appear

14. Motzkin, T.S., Straus, E.G.: Maxima for graphs and a new proof of a theorem of Túran. Can. J. Math. 17, 533-540 (1965)

15. Nesterov, Yu.: Random walk in a simplex and quadratic optimization over convex polytopes. CORE Discussion Paper 2003/71, CORE-UCL, Louvain-La-Neuve (2003)

16. Parrilo, P.A.: Structured semidefinite programs and semialgebraic geometry methods in robustness and optimization. PhD thesis, California Institute of Technology (2000)

17. Pólya, G.: Collected Papers, vol. 2, pp. 309-313. MIT Press, Cambridge, Mass, London (1974)

18. Powers, V., Reznick, B.: A new bound for Pólya's theorem with applications to polynomials positive on polyhedra. J. Pure Appl. Algeb. 164, 221-229 (2001)

19. Sherali, H.D., Adams, W.P.: A hierarchy of relaxations between the continuous and convex hull representations for zero-one programming problems. SIAM J. Discr. Math. 3: 101-112 (1992)

20. Zuluaga, L.F., Vera, J.C., Peña, J.F.: LMI approximations for cones of positive semidefinite forms. SIAM J. Optim. 16, 796-817 (2006) 